\title{
MPI as an Abstraction for Software-Hardware Interaction for HPRCs
}

\author{
Manuel Saldaña*, Arun Patel*, Christopher Madill ${ }^{\S}$, Daniel Nunes ${ }^{\dagger}$, Danyao Wang ${ }^{\dagger}$, \\ Henry Styles ${ }^{\ddagger}$, Andrew Putnam ${ }^{\ddagger}$, Ralph Wittig ${ }^{\ddagger}$, Paul Chow ${ }^{\dagger}$ \\ ${ }^{*}$ Arches Computing Systems, Ontario, Canada \\ Email:\{ms,ap\}@archescomputing.com \\ ${ }^{\dagger}$ University of Toronto, Department of Electrical and Computer Engineering, Ontario, Canada \\ Email:\{cmadill,dnunes,wangda,pc\}@eecg.toronto.edu \\ $\S$ University of Toronto, Department of Biochemistry, Ontario, Canada \\ Email:\{chris.madill $\} @$ utoronto.ca \\ $\ddagger$ Xilinx, San Jose, California, USA \\ Email:\{ralph.wittig, henry.styles, andrew.putnam\}@xilinx.com
}

\begin{abstract}
High Performance Reconfigurable Computers (HPRCs) consist of one or more standard microprocessors tightly coupled with one or more reconfigurable FPGAs. HPRCs have been shown to provide good speedups and good cost/performance ratios, but not necessarily ease of use, leading to a slow acceptance of this technology. HPRCs introduce new design challenges, such as the lack of portability across platforms, incompatibilities with legacy code, users reluctant to change their code base, a prolonged learning curve, and the need for a system-level Hardware/Software co-design development flow. This paper presents the evolution and current work on TMD-MPI, which started as an MPI-based programming model for Multiprocessor Systems-on-Chip implemented in FPGAs, and has now evolved to include multiple X86 processors. TMD-MPI is shown to address current design challenges in HPRC usage, suggesting that the MPI standard has enough syntax and semantics to program these new types of parallel architectures. Also presented is the TMD-MPI Ecosystem, which consists of research projects and tools that are developed around TMD-MPI to further improve HPRC usability.
\end{abstract}

\section{INTRODUCTION}

Portability in a typical High-Performance Computer (HPC) is achieved by using standards-based operating systems and software layers (Middleware) that abstract machine-specific hardware from the software application, as shown in Figure 1 on the left. The UNIX operating system (and its variants) and the MPI [1] library are examples of those de-facto standards that enabled portable parallel applications across HPCs. For HPRC designs to be truly portable, these concepts must be extended to the FPGAs, as they are now part of the application. Figure 1 shows how portability for HPRCs must come from two sides: application software and application hardware.

For HPRC's, the hardware operating system and hardware middleware should provide a standard abstraction for application hardware engines to access host-specific resources, such as external memory and communications. Examples of these host-specific communication resources are the X86-FPGA communication mediums, such as Intel's Front Side Bus (FSB) or QuickPath [2], AMD's HyperTransport [3], Cray's Rapid Array Transport [4], SGI's Scalable System Port-NUMA link connection [5], or even PCI Express. Companies such as Cray, SGI, Intel, Nallatech, XtremeData, DRC and SRC provide their own low-level software APIs and their own hardware interfaces for application hardware engines. HPRCs are now in a similar stage as HPCs were before the appearance of MPI [1] when every vendor had their own message-passing API to program their own supercomputers causing a lack of portable designs. Currently, there is no implemented standard API for a high-level parallel programming model that includes the interaction between X86 processors and FPGAs. There is, however, interesting progress done by the OpenFPGA[6] organization on this matter, which is compared against this work in Section V.

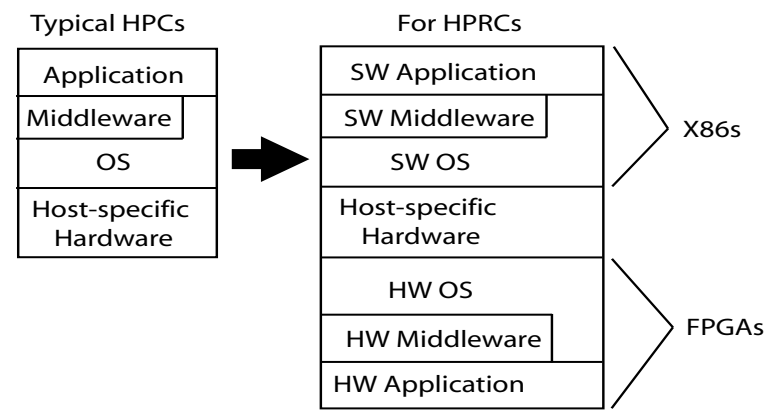

Fig. 1. Comparison between HPC and HPRC abstraction layers

Previous work proposed TMD-MPI [7][8][9][10] as a subset implementation of the MPI standard targeting Multiprocessor System-on-Chip implementations across multiple FPGAs to abstract hardware details from the user as well as to provide a well-known, high-level parallel programming API. In three years, TMD-MPI has evolved in a bottom-up approach to the point where it provides software and hardware middleware layers of abstraction for communications to enable the portable 
interaction between embedded processors, specialized hardware computing engines and now X86 processors; all programmed under the same message passing paradigm making it a unified programming model for HPRCs.

This paper provides a summary of TMD-MPI's philosophy and evolution, followed by a discussion of the new additions, current work and supporting tools and research projects called the "TMD-MPI Ecosystem". It is hoped that these research efforts generate discussion in the community regarding whether MPI is a good model for HPRCs or not, influencing perhaps existing MPI implementations to include FPGA support. It is time to consider how to include acceleration technologies, such as FPGAs, GPUs and CELL processors into a potential MPI3 accelerator-aware standard because accelerator technologies also have data movement and synchronization requirements.

The rest of the paper is organized as follows. Section II explains the motivations that led to the development and use of TMD-MPI. In Section III an updated version of the design flow based on recent work is presented. Section IV provides a real example of portability based on experiences using TMD-MPI. Section V contrasts TMD-MPI with related work regarding X86-FPGA communication. Section VI describes TMD-MPI's architecture and HPRC reference platform. In Section VII, the current functionality of TMD-MPI is described. Section VIII presents a synopsis of the projects that constitute the TMDMPI Ecosystem. Finally, some concluding remarks are given in Section IX.

\section{Motivation}

TMD-MPI was initially developed to address the need for a programming model for the TMD (Toronto Molecular Dynamics) machine being developed at the University of Toronto [10]. The TMD machine is a scalable MultiFPGA configurable system designed to accelerate computing intensive applications. The basic assumption is that FPGAs have enough resources to implement entire applications, as opposed to only parts of computations, such as small computing kernels. The objective is to have a tightly coupled mix of embedded processors (for control intensive parts of the application) and specialized hardware engines (to accelerate the compute-intensive parts of the application) all within the same FPGA or distributed among many FPGAs.

With multiple embedded processors and hardware engines (collectively referred to in this paper as computing elements, or CEs) interacting across multiple FPGAs, there is a need for a networking infrastructure and a distributed programming model. Most multi-FPGA systems have distributed memory (usually, each FPGA has its own external memory), and inside each FPGA there might be multiple CEs, each with their own local memory; hence, a programming model such as MPI seems adequate. With the appearance of tightly-coupled FPGAs and X86 processors sharing the main system memory [11], [12], [13], [14], [15], TMD-MPI has been extended to include communication with X86 processors, marking TMDMPI's entrance to the HPRC world.
Figure 2 shows the generic model of a HPRC machine. It is possibly a multi-host machine, where each host can have one or more X86 processors and one or more FPGAs, each processor with one or more X86 cores, and each FPGA with one or more computing elements inside. Note that in this model two or more FPGAs can indirectly share the same connection point to the host's internal interconnection system (FPGAs 1, 2 and 3 share a connection). TMD-MPI shows that the MPI standard is a reasonable abstraction for addressing the communication and synchronization of such architectures.

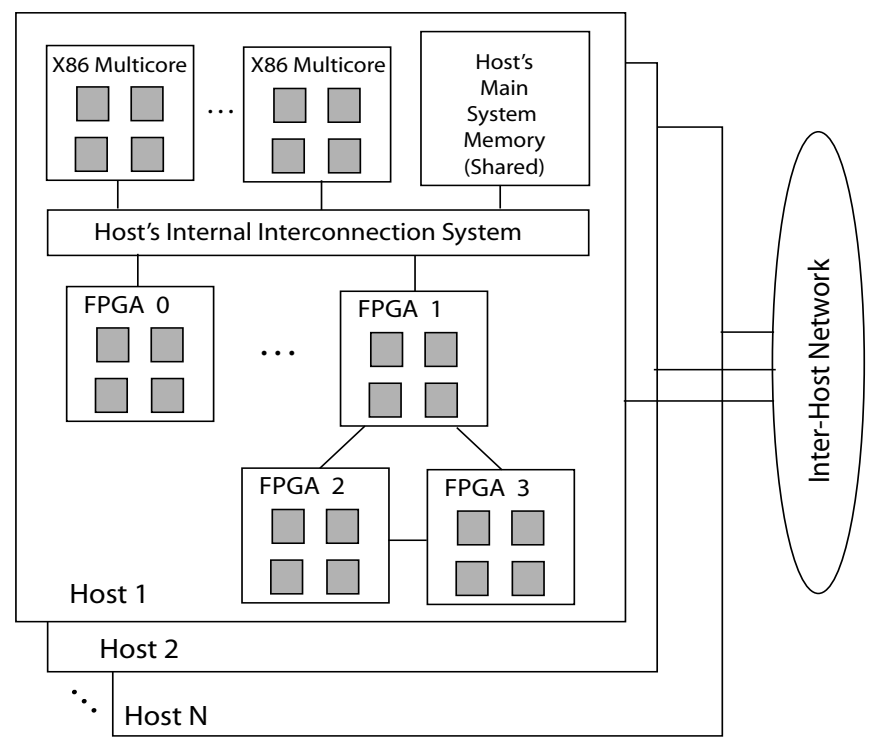

Fig. 2. HPRC generic model

There are two challenges when programming an application for this HPRC model. The first is the high-level, coarse-grain parallelization of the application, where decisions inherent to the parallel algorithm, such as data partitioning, synchronization and load balancing are made. The second challenge is the efficient use of the available FPGAs, where low-level decisions are taken, such as the number of pipeline stages, type of floating-point support and mapping a digital design to chip resources. A fully-automated flow for HPRCs that parallelizes an application and at the same time converts it into hardware is a double challenge. This is especially true if a non-parallelfriendly, non-hardware-friendly language is used, such as C.

Perhaps at some point projects on high-productivity parallel programming languages, such as Chapel [16] and X10 [17] can cooperate with tools that automatically generate HDL, such as ImpulseC [18], HandelC [19], Synfora [20], MitrionC [21], etc., to provide a fully-automated flow that overcomes the extraordinary challenges of programming HPRCs.

In the meantime, efficient applications for HPRCs have to be developed by a multidisciplinary team: a hardware engineer, a software engineer, and the application expert. The proposed approach is to deal with the two HPRC programming challenges independently. By parallelizing the code using MPI, the entire application is partitioned into smaller and simpler pieces of code, leaving the C-to-HDL tool (or hardware engineer) 
the easier task of turning into hardware more confined pieces of code. Writing high-level parallel code is still easier than writing low-level HDL code, so this approach is of great help to the non-hardware knowledgeable user. The experience of using TMD-MPI shows that MPI applied to FPGAs offers a convenient and clean interface between hardware designers and software developers that allows independent progress of both due to the standard interface and well-defined semantics. Pragmatically, HPRCs exist now and the user community cannot wait for fully automated tools to magically appear and make them trivial to use. TMD-MPI and its surrounding Ecosystem are examples of tools that exist today.

\section{Design Flow}

As previously discussed, the two major challenges to implementing an application on HPRCs are coarse-grain parallelization and HDL coding. To address these challenges, a topdown, step-by-step design flow was developed to implement applications using HPRCs. Figure 3 illustrates the updated version of the flow that was first introduced by Patel et. al. [10]. This new version includes the X86-FPGA interaction.

Step 1 begins by developing a prototype of the application in a high-level programming language such as $\mathrm{C} / \mathrm{C}++$. The resulting code is sequential in nature and is only intended to provide a prototype solution to the computing problem. At this stage, the application can be profiled to identify computationallyintensive routines. The results of this prototype design can be compared to the results of the final system and validate the correctness of the implementation at any step during the flow.

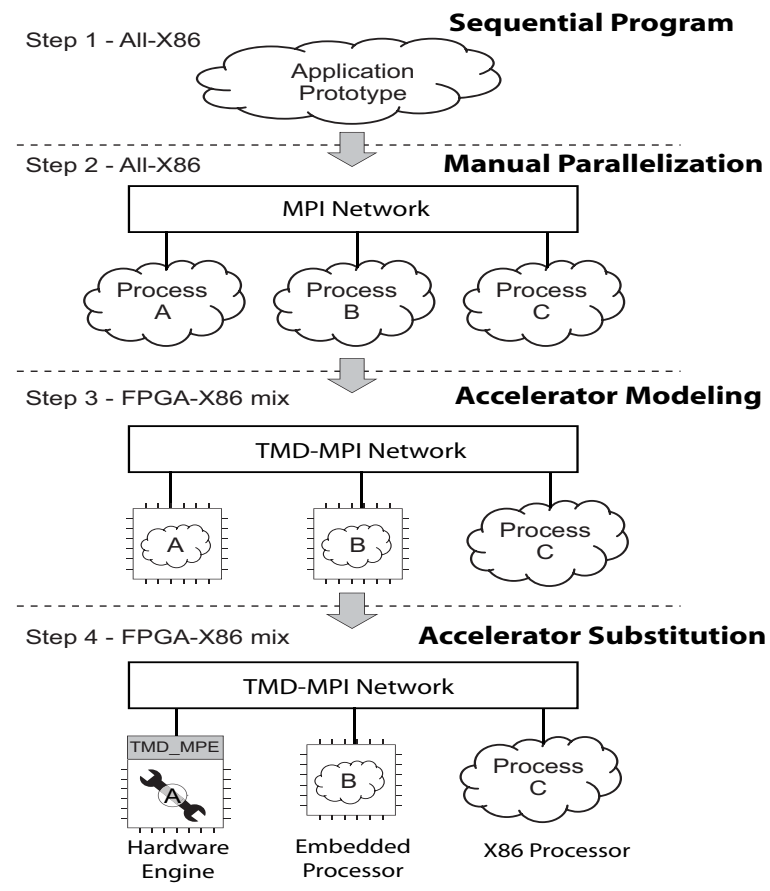

Fig. 3. Design Flow with TMD-MPI

Step 2 refines the prototype application by partitioning it into simple, well-defined processes that can be replicated to exploit the implicit parallelism of the application. At this point the user should have at least a hint about what processes are the most demanding, and therefore candidates for hardware engines. The user should avoid the use of any operating system calls or any feature that a hardware engine could not support. Inter-process communication is achieved using MPI, allowing the application to be developed and tested at the X86 level. No FPGA is involved yet. This approach has the advantage of allowing the programmer access to standard tools for developing, profiling and debugging. As an alternative to using TMD-MPI, other MPI implementations, such as MPICH [22] or OpenMPI [23] can be used, however, the programmer must use only the available MPI functions implemented in TMDMPI (see Section VII). Validation of the parallel algorithm can be done at this point, by comparing against the sequential version.

In Step 3, selected software processes developed in Step 2 are recompiled for embedded processors. The rest of the processes are still executed by the X86. The portability of MPI allows the software to be recompiled and executed on the embedded processors with the code practically unchanged. This is an optional intermediate step where hardware engines are emulated by embedded processors in the FPGA. At this stage, execution of the entire application using the FPGAs is possible. This allows the interaction between X86 processors, embedded processors and emulated computing engines to be tested and validated. This step gives the user the opportunity to better exploit communication locality by putting together in the same FPGA control-intensive processes (embedded processors) and compute-intensive processes (hardware engines) that have an intense communication pattern.

The final step of the programming flow substitutes algorithms executing on embedded processors with hardware engines. Translating the computationally-intensive processes into hardware engines is done manually in the current flow using typical FPGA design tools. However, since the system has already been partitioned into individual computing tasks and all communication primitives have been explicitly stated at this stage, C-to-HDL tools may also be used to perform this translation, or as discussed in Section VIII-C, MPI-to-HDL tools are feasible.

Once a computing engine has been designed, an additional message-passing engine (TMD-MPE, Section VI-E) is used to perform message passing functionality in hardware (e.g., protocol processing, handling message queues, divide large messages into packets), which simplifies the design of the computing engine. Special versions of the TMD-MPE can also be used by the embedded processors as a communication coprocessor enabling a more efficient data exchange.

\section{AN EXAMPLE OF PORTABILITY AND SCALABILITY}

This section gives an example of the portability that TMDMPI provides to a reconfigurable computing application being developed in the rapidly evolving field of FPGAs.

For the last three years, research has been conducted at the University of Toronto in collaboration with the Hospital 
for Sick Children to develop an FPGA-based machine to accelerate Molecular Dynamics (MD) simulations. These are atomic-level simulations of biomolecular systems used in biomedical research to design more effective drugs. The development of this machine began using the Amirix AP1000 PCI development boards [24]. Each board contains one XC2VP100 FPGA, 64 MB of external memory and four high-speed serial I/O links, which allowed five of these boards to be fully interconnected. The MD application coding was in progress when the new BEE2 platform [25] arrived with five XC2VP70 FPGAs per board, 4 GB of external memory per FPGA, using parallel LVDS lines and 18 high-speed serial links. This platform is obviously more integrated, provides more resources and potential for acceleration. Consequently, the development of the MD application continued on the BEE2 platform. The MD code was still in progress when the even newer Xilinx Accelerated Computing Platform (Xilinx ACP) arrived. This platform (further described in Section VI-A) has Virtex 5 FPGAs that can be connected directly to the Intel FSB by inserting them into one of the CPU sockets in the motherboard. The FPGAs are tightly coupled to X86 processors sharing 8 GB of memory, opening up more opportunities for acceleration. Currently, the development continues for this platform.

The question is how can one develop an application if the hardware keeps changing. Furthermore, how can existing code be isolated from future hardware changes. The impact of these hardware changes on the MD application has been minimized by using TMD-MPI. Indeed, there were changes, but all of them in the system infrastructure (what is described as SW/HW OS and SW/HW Middleware in Figure 1), not at the SW/HW application levels. The MD software code (written in $\mathrm{C}++$ ) and the MD hardware engines (HDL) have been mostly the same, changing only in their natural evolution towards a more complete MD simulator.

One of the necessary changes was to regenerate some of the core netlists to take advantage of the newer Virtex 5 architecture. Another change was computing element reallocations. The bonded and non-bonded force calculations (particular types of forces in MD) were performed by two different computing elements that were located in the same FPGA, but when switching from the AP1000 boards to the BEE2 boards the bonded forces compute engine had to be reallocated to another FPGA because the BEE2 board has smaller FPGAs than the AP1000 board. Moreover, the bonded force computing element was originally a PowerPC processor, then it became a MicroBlaze soft-processor, but most likely it will end up being an X86 processor. Through all these changes, TMD-MPI provided tolerance to hardware changes and made it possible to perform architectural explorations and propose changes to achieve better performance without impacting the MD application code-base.

Another advantage of using TMD-MPI was the abstraction of the FPGA configuration process, which is usually not as easy to run as a typical program, and it is also a completely different procedure between the AP1000 boards, the BEE2 boards and the Xilinx ACP. Furthermore, in a HPRC, starting the execution of a large number of X86 software processes and configuring a large number of FPGAs must be automated. By using mpirun/mpiexec scripts, a difference like this can be minimized and hidden from the user perspective.

\section{RELATED WORK}

TMD-MPI was created with the influence of the Multiprocessor System-on-Chip [26] and Network-on-Chip research fields. Related work of those areas to TMD-MPI is discussed in previous work [8][9] and briefly mentioned in Section VI-B. In this Section, current APIs for X86-FPGA communication are compared with TMD-MPI.

Currently, the most common usage of MPI on HPRCs is at the host level [27], where X86 processors exchange messages using MPI and then forward the received data to the FPGAs, which is a mere slave to the $\mathrm{X} 86$ processor. This uses the $\mathrm{X} 86$ as a message relay introducing latencies and complicating the programming model because the user has to be aware of two different APIs: one for message-passing and another for interacting with the FPGA. This section shows how MPI's high-level API can be used instead of low-level API calls to perform X86-FPGA communications, with the added benefit of a broader communication context, more abstraction and a familiar API, providing a unified programming model. Note that many features that are available in the MPI standard are not implemented in TMD-MPI yet, and that certain features are not defined in the MPI standard but left open to the implementation. Here references to MPI mean the API itself, and references to TMD-MPI means our particular implementation of MPI.

At the time of writing this paper, OpenFPGA has released its first General API Specification 0.4 (GenAPI) draft [28]. Its objective is to propose an industry standard API for highlevel language access to reconfigurable computing resources in a portable manner. The draft is an excellent compilation of common X86-FPGA communication requirements for HPRCs. GenAPI shares practically all the same design goals as TMDMPI, however, there is a fundamental difference: the scope of the standard. GenAPI only focuses on the low-level X86FPGA interaction, not making any assumptions on higher level forms of communication, such as FPGA-initiated transfers, inter-host communications, inter-FPGA communications and intra-FPGA communications. In contrast, the MPI standard is agnostic of the communication media and requires that every rank (a task in the MPI jargon) be able to initiate transfers (Master) at any time regardless of its physical location. In this sense, the FPGA is no longer a passive slave in the system.

Similar to GenAPI, HPRC vendors provide their own APIs, but with the same limited scope: X86-FPGA communication, which is a subset of the communications that TMD-MPI is able to provide. Table I summarizes these communication capabilities. TMD-MPI will be able to provide communications at the host-to-host level, X86-to-X86 level, FPGA-to-FPGA level and intra-FPGA level (CE to CE); and in between these categories as well. That is, a hardware engine inside an FPGA in one host can exchange data with another hardware engine 


\begin{tabular}{|l|l|}
\hline Master & Slave \\
\hline \hline X86 (core) & X86 (core) \\
\hline X86 (core) & FPGA (CE) \\
\hline FPGA (CE) & X86 (core) \\
\hline FPGA (CE) & FPGA (CE) \\
\hline Host A (X86 core or CE) & Host B (X86 core or CE) \\
\hline
\end{tabular}

TABLE I

TMD-MPI'S COMMUNICATION CAPABILITIES

inside another FPGA in a remote host, without the need of an X86 to start or negotiate the communications.

In TMD-MPI, because of its on-chip origins and its distributed nature, achieving multi-level communications is straightforward. However, to make TMD-MPI portable across HPRC platforms, the low-level APIs are needed exclusively for X86-FPGA communication. For example, on the Xilinx ACP platform a custom low-level API is being used, but in principle other APIs could be used, such as Intel's AAL [29]. The GenAPI standardization efforts would help TMD-MPI by not having to implement a different version for each HPRC lowlevel API. By looking at some HPRC vendor APIs [4][5][29] and GenAPI, four general categories can be distinguished in the functions available: Initialization and termination, FPGA management, data transfer, and memory allocation. The APIs are driver-like interfaces to the FPGA (open, close, write, read, mmap, etc.), but a system-level design of parallel applications that include FPGAs, needs a higher-level of abstraction.

Some of the low-level API calls can be easily mapped to MPI calls. For example, MPI function MPI_Init() is used to initialize internal data structures, to open the FPGA device and to signal the FPGA to start running. The opposite, MPI_Finalize() is used to signal the end of the application to the FPGA, remove the internal structures and close the FPGA device. MPI_Send(), MPI_Isend(), MPI_Recv() and MPI_Irecv() are used to send/receive data to/from the FPGA in a blocking and non-blocking manner. For memory allocation, MPI provides the MPI_Alloc_mem() and MPI_Free_mem() functions to allocate/deallocate memory that the FPGA can access. An MPI example using some of these functions is shown in Section VI-C.

Of course the MPI standard does not define how to download a bitstream to an FPGA, but this can be left open to the implementation. Currently in TMD-MPI, downloading a bitstream is part of the mpiexec or mpirun commands. A similar idea on configuring distributed FPGAs and a unified programming model was proposed by Jones et al. [30], but the authors use their own API whereas our objective is to use the standard MPI API itself. Also, TMD-MPI has a broader scope as it includes more communication modes as shown in Table I.

Another interesting effort trying to bring portability to HPRCs is Vforce [31]. The authors provide a high-level object-oriented framework based on the VISPL++ standard, which is a vector and image processing library API. Their approach is quite similar to ours in two fundamental points.
First, one layer in their framework interacts with the lowlevel API calls rather than the final user's code, insulating the application from hardware changes. TMD-MPI uses a low-level driver to access the FPGA, not the user's code, which uses MPI calls. The second similarity is that they allow domain experts, such as FPGA designers, to create specialized hardware library components that can be interfaced with their framework. MPI allows the creation of third-party parallel libraries, and with TMD-MPI it is possible to have software-hardware parallel libraries that interact based on an application-level message-passing protocol.

\section{TMD-MPI ARCHITECTURE}

In this section the Xilinx ACP is first presented as an example of the HPRC model described in Section II. Then the mechanism for TMD-MPI data transfers in the ACP using a mix of shared and distributed memory is explained.

\section{A. HPRC Implementation Platform}

TMD-MPI has been used in a variety of platforms, but the focus here will be on the Xilinx ACP as it is the newest platform and current target.

The base system is an instance of the HPRC model shown in Figure 2. It is an Intel quad-socket Server System, the S7000FC4UR. One of the processor sockets has an Intel Quad-core Xeon 7300 processor and the other socket has the Xilinx ACP. Currently, the remaining sockets are not used, but more X86 processors or Xilinx ACPs could be added. The Xilinx ACP consists of a stack of up to three PCB layers (modules). The first layer (bottom-up) contains the bridge FPGA (XC5VLX110) and is physically placed in one of the $\mathrm{X} 86$ sockets on the motherboard. Its function is to provide a connection point to Intel's FSB and provide communications for the upper-level layers. The second and third layers are known as dual-compute FPGA modules. Each module contains two XC5VLX330 FPGAs in the same PCB. All the FPGAs are directly connected by LVDS lines in the same layer and between layers. The Xilinx ACP platform and the X86s can share up to $64 \mathrm{~GB}$ of RAM through the FSB, and the machine is running a standard SMP Linux distribution. In this paper this machine is used as a reference implementation platform for TMD-MPI, but most of the concepts can be applied to other HPRCs as long as the FPGAs have access to main system memory.

\section{B. Intra-FPGA and Inter-FPGA communication}

A generic example for a multi-FPGA board is shown in Figure 4. The figure could physically represent five AP1000 boards, one BEE2 board or one Xilinx ACP platform with one bridge FPGA and two dual-compute FPGA modules. The group of FPGAs is called an FPGA_cluster. The communication is logically divided in Tiers based on its scope.

Tier 1 is the on-chip network that is used to exchange data between multiple computing elements within the same FPGA. This network is a set of point-to-point channels (a pair of FIFOs, one for transmission, one for reception), usually 
in a fully-connected topology, but other topologies can be used as well, depending on the communication needs. The network latency is usually a few clock cycles depending on the topology. The bandwidth is limited by the FPGA frequency and the width of the point-to-point channels.

The Tier 2 network is used for inter-FPGA communication within the same FPGA_cluster. The gateways are connection points (bridges) between Tier 1 and Tier 2. Usually this network uses high-speed serial links or parallel LVDS lines. An important requirement is that this network has CRC and packet retransmission that guarantees error free transfers. One of the FPGAs, has a special gateway that connects Tier 2 to Tier 3 and Tier 4.

Tier 3 , not shown in the picture, would be all the communications within the same host: FPGA_cluster-X86, X86X86, and FPGA_cluster-FPGA_cluster. Finally, Tier 4, also not shown in the figure would be the host-host communication.

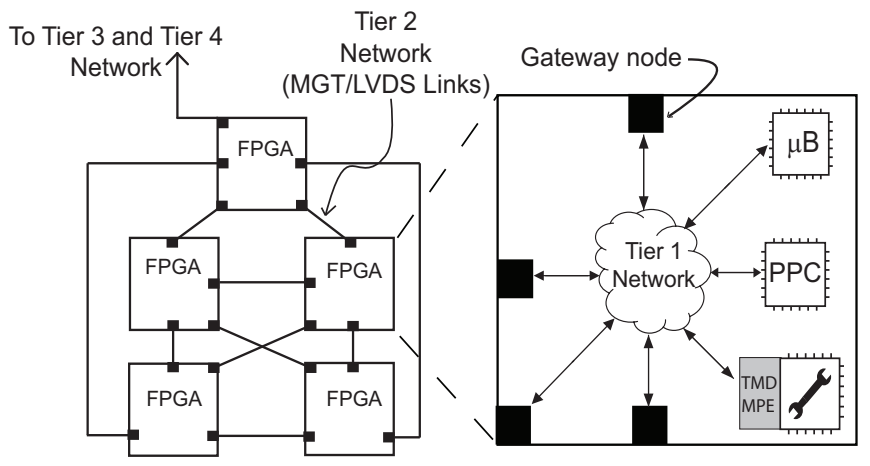

Fig. 4. Inter-FPGA and Intra-FPGA communication

The Tier 1 network uses small distributed on-chip network interfaces (NetIfs), that forward packets to the right channels based on a routing table. The NetIfs use a buffer-less cutthrough routing approach to reduce latency and to save on-chip resources. Some degree of buffering is implicit by using FIFOs as channels. Since all communication is FIFO-based, full and exists signals are used for flow control back-propagating the full condition to the sender.

Figure 5 shows an example of a connection between two computing elements: a MicroBlaze in FPGA 1 and a hardware engine in FPGA 2. Tier 1 and Tier 2 networks might have different protocols, in which case the Bridge block will handle network packet translations as they pass through the bridge. The bridge block can be as simple as just wires when packet formats between Tier 1 and Tier 2 are the same. However, in the case of FPGA_cluster-X86 communication the bridge is considerably more complicated, as will be seen Section VI-D.

Finally, the Off-chip Communication Controller (OCCC) is the block handling the physical access to the communication media (MGTs, LVDS, FSB, etc.). This block delivers errorfree data to the FPGA from the outside world and vice-versa. In the case of the AP1000 boards, this block is the FSL_Aurora core [32], which uses the Xilinx MGT and Aurora link layer protocol for serial I/O. In the case of the BEE2, the blocks used are the FSL_interchip core for the LVDS interface (parallel link) and the FSL_XAUI core for the 10Gbit XAUI interface (serial link). In the case of the Xilinx ACP platform, the block is the Intel-Xilinx FSB protocol hardware core, which allows the FPGA to access the host's shared memory.

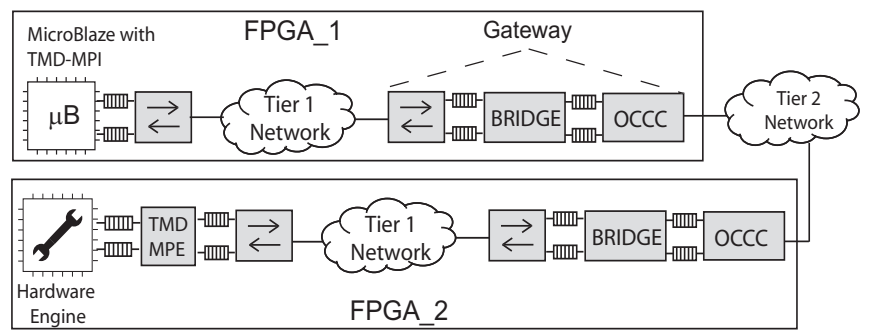

$\rightleftarrows \quad$ Network Interface (Netlf) $\quad-$-III- FIFO

Fig. 5. Inter-FPGA and Intra-FPGA communication

\section{Shared memory communication}

TMD-MPI requires a distributed memory mind set from the user, but it takes advantage of the shared memory to perform the message passing for X86-X86, FPGA-X86 and X86-FPGA communication in the same host. TMD-MPI provides two ways of performing shared memory transfers. One is the typical approach that performs an extra copy from the user source buffer to an intermediate shared buffer, and lets the receiver copy the data from the intermediate buffer to the user destination buffer. The second approach available in TMDMPI is a zero-copy technique, in which the sender or the receiver can copy the data directly from the user source buffer to the user destination buffer, without incurring the extra copy to the intermediate buffer.

TMD-MPI will automatically select one or the other based on how the buffer is created. If the source or destination (or both) buffers are allocated using the MPI_Alloc_mem() function then MPI will automatically select the zero-copy approach, otherwise TMD-MPI will use the intermediate buffers. This works for both X86-X86 and FPGA-X86 communication. However, it does not mean that the user must allocate a buffer to be able to transfer it to the FPGA or to the X86. It just means that one way will be faster than the other depending on the situation.

This is convenient because at the programming model level the user does not have to allocate every buffer to be sent, even if it is a small payload. This is exemplified in Figure 6. The user can declare a variable or an array in a typical way and just send them (the extra copy approach will be selected by TMDMPI), or the user can allocate two buffers and send them (zerocopy will be selected by TMD-MPI). A zero-copy transfer is convenient when transferring large amounts of data, usually critical to the performance of the application and justifying a memory allocation. But a simple intermediate copy would suffice for a non-critical small transfer, or when it is necessary to transfer large data sets at initialization stages, again not critical for performance; otherwise, extra memory allocations 
might be tedious for the programmer. In Figure 6, the variable dest can be the rank of an X86 processor, a MicroBlaze, a PowerPC or a hardware engine. The same API is used.

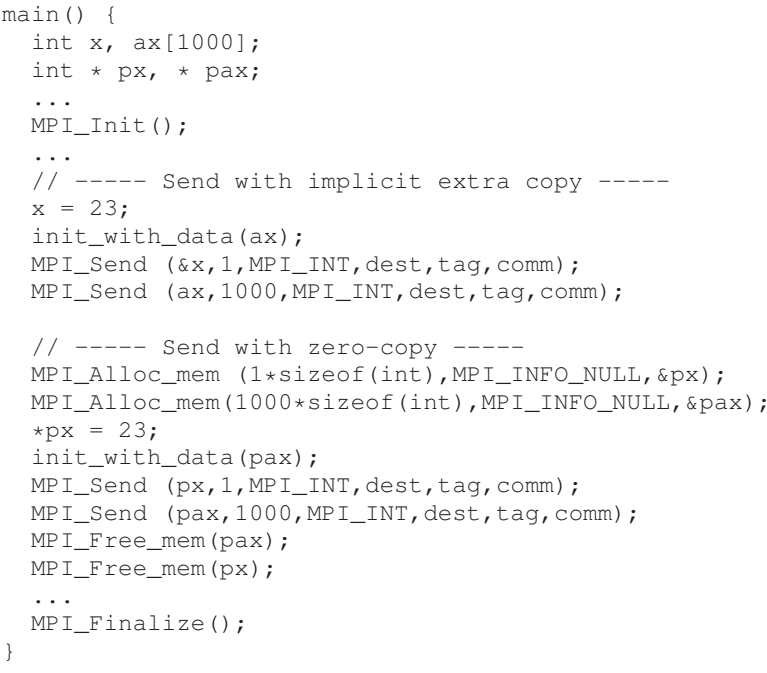

Fig. 6. Transfers with implicit extra copy and zero-copy

\section{The MPI_FSB_Bridge}

The communication at the X86-X86 level is achieved using shared memory, and the computing elements inside the FPGA exchange messages using the Network-on-Chip. To exchange messages between X86s and computing elements, the data must travel through a shared memory MPI bridge (MPI_Bridge), which implements in hardware the same shared memory protocol that the X86 processors use, as shown in Figure 7. This bridge takes data to/from the NoC and issues read or write memory requests to the vendor-specific low-level Off-Chip Communication Controller (OCCC), which executes the request. The MPI_Bridge effectively abstracts the vendorspecific communication details from the rest of the on-chip network. In this way, the FPGA design can be ported as long as there is a corresponding MPI_Bridge for the new HPRC. Other interfaces, such as external memory might still be an impediment to porting designs from one HPRC to another.

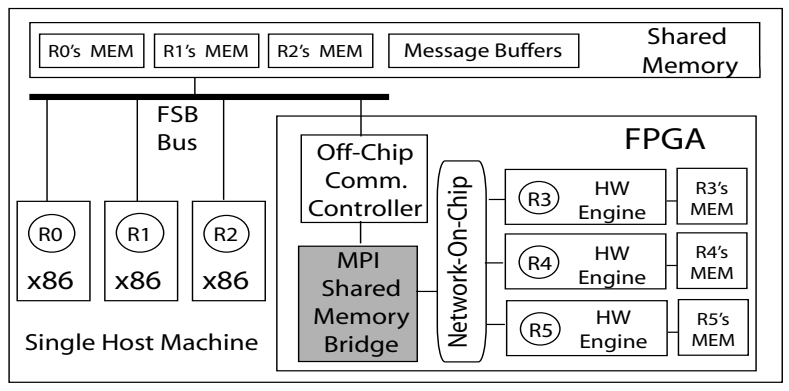

Fig. 7. The MPI Shared Memory Bridge is the interface between the shared memory and the Network-on-Chip.

For this paper, the Intel FSB is used as the communication medium but the same concepts can be applied to other communication media, such as AMD's HyperTransport [3], Intel's QuickPath [2], Cray's Rapid Array Transport [4], SGI's Scalable System Port-NUMA link connection [5], or even with a standard PCI Express core because they all provide a physical connection to the main system memory. The communication medium determines what OCCC to use, and in this case the Intel-Xilinx FSB communication core handles the low-level protocol to read and write to memory as well as the memory coherence control. But most importantly, TMDMPI's shared memory message-passing protocol should be the same across HPRCs or with minor variations. The only change is the physical interconnection between the MPI_Bridge and the vendor-specific OCCC. By implementing a MPI_Bridge for each type of OCCC, the system is portable. For example, in this paper a MPI_Xilinx_FSB_Bridge is used, but an MPI_Cray_Bridge could be implemented to use a Cray HPRC.

An extension of this approach to a multi-host distributed memory machine is natural since message-passing assumes no shared memory. In this case, an MPI_Ethernet_Bridge could be used, or any other point-to-point communication interface, to allow the connection of multiple hosts through the FPGAs.

\section{E. TMD-MPE}

For processors, TMD-MPI is a software library that provides the message passing capabilities, but for hardware engines to have message passing capabilities they must use a block called TMD-MPE (TMD-Message Passing Engine), which encapsulates in hardware some of the TMD-MPI functionality. The TMD-MPE provides the hardware equivalent to MPI_Send and MPI_Recv to a computing element in the FPGA, handles unexpected messages and the communication protocol, and divides large messages into smaller size packets to be sent through the network. As shown in Figure 8, the TMD-MPE is connected between the computing element and the onchip network interface (NetIf). The TMD-MPE receives the message parameters and data from the computing element, such as the operation (whether it is sending or receiving a message), the destination node id (rank of the process in the MPI environment), the length of the message, and an identification number for the message (the tag parameter in a normal MPI send/receive operation). After this, the TMD-MPE will handle the communications through the network with the destination rank. The interfaces between the TMD-MPE and the computing element are four FIFOs (two for commands and two for data) making integration easy. These FIFOs can be asynchronous FIFOs allowing the computing elements to operate at different frequencies than the rest of the system.

The main application pipeline, or computing engine is wrapped inside a block that has a user-defined state machine whose purpose is to issue send and receive commands using the command FIFOs. The actual data should pass through the data FIFOs to and from the application pipeline. In this way, communications and computation are cleanly separated. The synchronization between the state machine and the application pipeline can be achieved through user defined simple control signals, such as busy or done signals, or even register values 
that may act as input parameters to the application pipeline or return codes from the application pipeline that may change the communication pattern.

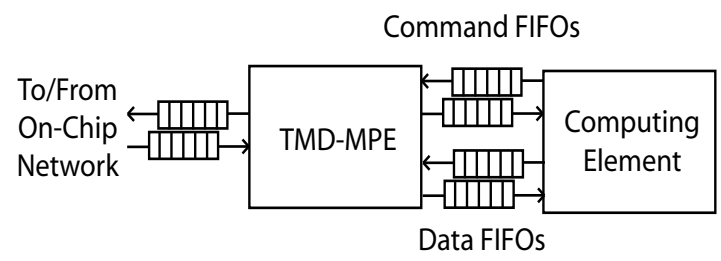

Fig. 8. Connection of a the application hrdware and the TMD_MPE.

\section{ViI. CURRent Functionality}

TMD-MPI is in constant development because the MPI standard is very broad and there are still many features in the standard that are not implemented in TMD-MPI. Additionally, there are requirements in HPRCs that are not addressed in the standard, and some additions, such as the bitstream configuration and software resets to FPGAs, are functions that need to be placed somewhere during the execution of an MPI program. Our policy to expand TMD-MPI is on an as-needed basis. The current functions in TMD-MPI are listed in Table II.

TABLE II

FUNCTIONALITY OF TMD-MPI SOFTWARE LIBRARY

\begin{tabular}{|c|c|}
\hline MPI_Init & Initializes TMD-MPI environment \\
\hline MPI_Finalize & Terminates TMD-MPI environment \\
\hline MP I_Comm_rank & Get rank of calling process in a group \\
\hline MPI_Comm_size & Get number of processes in a group \\
\hline MP I_Wtime & $\begin{array}{l}\text { Returns number of seconds elapsed since } \\
\text { application initialization }\end{array}$ \\
\hline MPI_Send & Sends a message to a destination process \\
\hline MPI_Recv & Receives a message from a source process \\
\hline MP I_Isend & Non-blocking send \\
\hline MPI_Irecv & Non-blocking receive \\
\hline MPI_Issend & $\begin{array}{l}\text { Non-blocking send using synchronous pro- } \\
\text { tocol }\end{array}$ \\
\hline MPI_Test & Tests if a non-blocking request is complete \\
\hline MPI_Wait & $\begin{array}{l}\text { Blocks the calling process until a non- } \\
\text { blocking request is complete }\end{array}$ \\
\hline MPI_Waitall & $\begin{array}{l}\text { Blocks the calling process until all the non- } \\
\text { blocking requests in an array are complete }\end{array}$ \\
\hline MPI_Barrier & Synchronizes all the processes in the group \\
\hline MPI_Bcast & $\begin{array}{l}\text { Broadcasts message from root process to } \\
\text { all other processes in the group }\end{array}$ \\
\hline MPI_Reduce & $\begin{array}{l}\text { Reduces values from all processes in the } \\
\text { group to a single value in root process }\end{array}$ \\
\hline MPI_Allreduce & $\begin{array}{l}\text { Reduces values from all processes in the } \\
\text { group, and broadcast the result to all the } \\
\text { processes in the group }\end{array}$ \\
\hline MPI_Gather & Gathers values from a group of processes \\
\hline MPI_Alloc_mem & $\begin{array}{l}\text { Allocates memory for Remote Memory } \\
\text { Access (zero-copy transfers) }\end{array}$ \\
\hline MP I_Free_mem & $\begin{array}{l}\text { Deallocates memory that was reserved us- } \\
\text { ing MPI_Alloc_mem }\end{array}$ \\
\hline
\end{tabular}

Among other restrictions, the TMD-MPE only implements point-to-point send and receive, no collective operations. In software, TMD-MPI collective operations can only perform
MPI_SUM and MPI_MAX on scalar values. There is only one communicator (MPI_COMM_WORLD) and only the rendezvous message-passing protocol is implemented (no eager protocol for scalability reasons). However, some of these limitations can be relatively easy to overcome, and they are scheduled to be done in the TMD-MPI project timeline.

\section{TMD-MPI ECOSYSTEM}

There is ongoing research at the University of Toronto to create a supporting framework around TMD-MPI. The objective is to facilitate the application development for HPRCs by providing automation, debugging, verification and profiling tools. In this section, the goals and status for each of these projects is briefly summarized.

\section{A. Profiling TMD-MPI}

In a parallel application, it is very important to understand how much time an application spends in communication and computation to identify potential improvements. Situations, such as communication bottlenecks and workload imbalance can be detected by profiling the application. The MPICH distribution includes the MPI Parallel Environment (MPE) [33], which provides the ability to profile different sections of the user application code as well as the MPICH code itself. This is done by code instrumentation using a predefined profiling API. The MPE includes a tool called JumpShot [34] that visualizes the profiler logs, allowing a user to see what each rank is doing over a common timeline.

Inspired by this, a similar profiler for TMD-MPI was implemented, which works with hardware computing elements in FPGAS. The TMD-MPI profiler [35] connects hardware blocks called tracers to the TMD-MPE, which provides all the information needed to profile the message-passing activity over the TMD-MPI network. A similar tracker is attached to a hardware engine or to an embedded processor to log computing events, such as pipeline busy signals that can help to determine the hardware engine utilization. The logs created by the TMD-MPI profiler are compatible with the logs created by the MPE allowing the same JumpShot GUI to be used; therefore, reducing the learning curve by not asking the user to learn a different tool, if already familiar with JumpShot. In the case of processors, the TMD-MPI profiler uses part of the same profiling API as the MPE.

Figure 9, is an example of the JumpShot GUI reporting data gathered by the TMD-MPI profiler. It shows a nonblocking data exchange between eight computing engines, implemented in an FPGA. The horizontal bars represent the time to receive a message (hash bar), compute (solid bar) and send a message (checker bar), and the arrows are control messages in the rendezvous protocol (request to send, clear to send and the payload data transfer). An overlap can be seen between communications and computation (bars with diagonal lines), which is not seen in the blocking version (not shown). For more details on this particular project see the work presented by Nunes et. al. [35]. 
The TMD-MPI profiler has been implemented and tested only on the BEE2 platforms, and is able to use multiple boards. Future work for this project includes adding support for the X86-FPGA interaction.

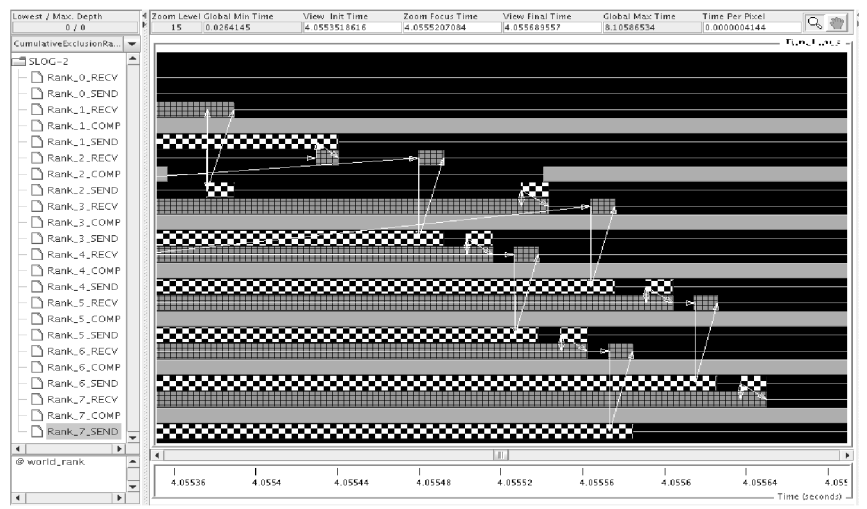

Fig. 9. Jumpshot GUI showing data gathered using the TMD-MPI profiler

\section{B. Message Passing Simulation Framework}

Compared to profiling, simulation is usually performed at the initial stages of the development to get a working FPGA-based system. A profiler is used to help improve the performance based on long-running, full-speed tests with real data sets, whereas a simulator is used to develop a functionally correct system, and run tests with size-reduced data sets at simulation speed. A simulation allows full visibility into the FPGA design and a fast code-debug-recompile cycle because there is no place-and-route process after design modifications.

In the case of HPRCs, there are two components: the X86 software and the FPGA hardware. To design, debug and verify such architectures, co-design techniques are taken from the embedded world but applied to the HPC world. These techniques are based on a system-level view of the application, and focuses on the interaction between hardware and software simultaneously, rather than designing two independent entities that will not coordinate efficiently. For this reason, the Message-passing Simulation Framework (MSF) [36] was created, which allows X86 software processes (R0 and R1 in Figure 10) to exchange MPI messages with the computing elements implemented in the FPGA (R2 and R3), which is being emulated by a simulator.

In a typical MPI parallel program, an MPI rank is not tested in isolation from the other MPI ranks. It has to be tested with all ranks running at once to verify the correct collective operation and synchronization between them. With FPGAs as containers of MPI ranks, they must be part of the system-level testing process. The MSF's objective is to test and debug userdefined MPI-based application-level protocols as well as the correct functionality of hardware engines, all at once.

Figure 10 shows how the FPGA is being simulated using ModelSim [37], but from the X86 processor perspective it appears like a slow FPGA (simulation speed). The key element in the MSF is the use of Modelsim's Foreign Language Interface (FLI), which allows a $\mathrm{C}$ program to have access to
Modelsim's simulation information, such as signals, register values and simulation control parameters. The MSF FLI module ( $\mathrm{C}$ code) replaces the vendor-specific OCCC (explained in Section VI-D) by providing the required functionality directly to the MPI Shared Memory Bridge. The MSF FLI accepts the MPI bridge memory requests (address and data) and performs the reads and writes directly to the shared memory buffers.

The MSF has been used to develop and debug the TMDMPI infrastructure itself, but it can also be used in applications as well. A simulation of a LINPACK benchmark core example interacting with X86s and more details on this project can be found in the work presented by Saldaña et. al. [36].

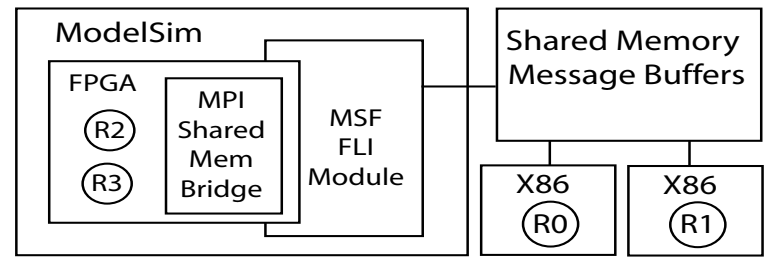

Fig. 10. The MSF FLI module in a HPRC

\section{Not just C-to-HDL, but MPI-to-HDL}

Currently, much research is being done on C-to-HDL tools because they will allow software developers, with no knowledge of hardware, to create FPGA-based hardware accelerators. However, this field is challenging because of the complexities of modern software and algorithms. A research project at the University of Toronto focuses on mixing MPI and a C-to-HDL tool (Synfora's PICO Express FPGA [20]). The resulting tool flow is called AUTO [38]. The objective is to take an MPI program written in $\mathrm{C}$ as input and produce a hardware computing engine that can be interfaced with the TMD-MPE allowing it to be part of the TMD-MPI network.

The basic assumptions are that a parallel MPI code (at least for the rank that is a candidate to be turned into hardware) is simpler than the entire sequential application to be parallelized, and that explicit statement of the communication requirements (MPI_Send() and MPI_Recv() function calls) can be easily converted to stream-like reads and writes to FIFOs. Remember from Section VI-E that FIFOs are used as interfaces to the TMD-MPE, which takes care of the message-passing protocol.

Figure 11, shows the AUTO flow. First the MPI code input is run through a preprocessing script that inserts codes that translate MPI function calls to stream operations in addition to hints and directives into a copy of the source code. The annotated code is then passed to PICO Express (Synfora's compiler), which translates it into verilog hardware files. These files are used by a packing script, which adds some control logic code and returns a complete pcore directory structure and files ready to be integrated into the Xilinx EDK tool.

A hardware engine was created from a simple MPI program that computes the Jacobi Iterations method to solve the Heat Equation using the AUTO flow. Results show that PICO Express works well for a subset of the $\mathrm{C}$ language; future additions to support pointers, more flexible looping structures, 
and floating-point will be welcome. An MPI program as a superset of a $\mathrm{C}$ program also suffers from the same lack of features. However, the extra work required to parse the MPI part over a plain $\mathrm{C}$ program is feasible. At this moment, the flow is designed to use Synfora, but a similar approach could be applied to ImpulseC, Handel-C, Mitrion-C, or any other Cto-HDL tool. See Wang [38] for more details on this project.

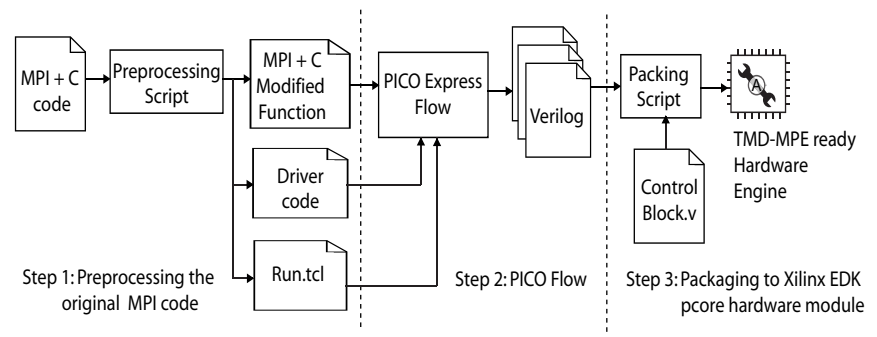

Fig. 11. MPI-to-HDL using the AUTO flow

\section{CONCLUSION}

In this paper, the ideas that support MPI as a feasible programming model that abstracts the communications in a HPRC have been presented. The fact that MPI is based on a wellknown and successful standard makes it even more appealing for parallel software developers. In the case of HPRCs, MPI can benefit from OpenFPGA's API standardization efforts, it can use C-to-HDL tools and encourage its improvements, and nothing prevents the use of a mixed shared memory plus MPI programming approach, if that helps to raise the programming abstraction. MPI provides a standard API for HPRCs that promotes portability across HPRCs, a common programming model for both software and hardware and easy scalability of the application.

TMD-MPI has been developed as a proof-of-concept implementation. Although it is still work in progress, it is currently being used to code an MD application in an HPRC. A future article will discuss TMD-MPI performance measurements as it requires more in depth analysis.

\section{ACKNOWLEDGMENT}

We acknowledge the CMC/SOCRN, NSERC and Xilinx for the hardware, tools and funding provided for this project.

\section{REFERENCES}

[1] The MPI Forum, "MPI: a message passing interface," in Supercomputing '93: Proceedings of the 1993 ACM/IEEE conference on Supercomputing. New York, NY, USA: ACM Press, 1993, pp. 878-883.

[2] Intel, "Intel Quick Path Architecture (White Paper)," http://www.intel. com/technology/quickpath/whitepaper.pdf [Accessed: September, 2008].

[3] HyperTransport Consortium, http://www.hypertransport.org.

[4] Cray Inc., CRAY XD1 FPGA Development, 2005, pp. 9-11, pp. 63-66.

[5] SGI, Reconfigurable Application-Specific Computing Users Guide, Jan 2008, pp. 9-12, pp. 223-244.

[6] “OpenFPGA," http://www.openfpga.org/.

[7] Manuel Saldaña, "A Parallel Programming Model for a Multi-FPGA Multiprocessor Machine," Master's thesis, University of Toronto, 2006.

[8] M. Saldaña and P. Chow, "TMD-MPI: An MPI Implementation for Multiple Processors Across Multiple FPGAs," in Proceedings of the 16th International Conference on Field-Programmable Logic and Applications, Madrid, Spain, 2006.
[9] M. Saldaña, D. Nunes, E. Ramalho, and P. Chow, "Configuration and Programming of Heterogeneous Multiprocessors on a Multi-FPGA System Using TMD-MPI," in In the Proc. of the 3rd Intl. Conf. on Reconfigurable Computing and FPGAs., San Luis Potosi, Mexico, 2006.

[10] A. Patel, C. A. Madill, M. Saldana, C. Comis, R. Pomes, and P. Chow, "A Scalable FPGA-based Multiprocessor," Field-Programmable Custom Computing Machines, 2006. FCCM '06. 14th Annual IEEE Symposium on, pp. 111-120, April 2006.

[11] Cray, Inc., "Cray XD1 Supercomputer for Reconfigurable Computing," http://www.cray.com/downloads/FPGADatasheet.pdf [Accessed: Sept. 2008].

[12] Nallatech, Inc., http://www.nallatech.com/.

[13] SRC Computers, Inc., http://www.srccomp.com/

[14] DRC computer, http://www.drccomputer.com/.

[15] Xtreme Data Inc., http://www.xtremedatainc.com/

[16] D. Callahan, B. Chamberlain, and H. Zima, "The Cascade High Productivity Language," Proc. Ninth Intl. Workshop on High-Level Parallel Programming Models and Supportive Environments., pp. 52-60, April 2004.

[17] Ebcioglu, Saraswat, and Sarkar, "X10: an Experimental Language for High Productivity Programming of Scalable Systems," Productivity and Performance in High-End Computing (P-PHEC). Second International Workshop on, February 2005.

[18] Impulse Accelerated Technologies, Inc., http://www.impulsec.com.

[19] Celoxica, LTD., http://www.celoxica.com.

[20] Synfora, Inc., http://www.synfora.com.

[21] Mitrionics, Inc., http://www.mitrionics.com.

[22] W. Gropp, E. Lusk, N. Doss, and A. Skjellum, "A High-Performance, Portable Implementation of the MPI Message Passing Interface Standard," Parallel Computing, vol. 22, no. 6, pp. 789-828, Sept. 1996.

[23] R. L. Graham, T. S. Woodall, and J. M. Squyres, "Open MPI: A Flexible High Performance MPI," in Proceedings, 6th Annual Intl. Conf. Parallel Processing and Applied Mathematics, Poznan, Poland, Sept. 2005.

[24] Amirix Systems, Inc., http://www.amirix.com/

[25] C. Chang, J. Wawrzynek, and R. W. Brodersen, "BEE2: A High-End Reconfigurable Computing System," IEEE Des. Test '05, vol. 22, no. 2, pp. 114-125, 2005.

[26] A. Jerraya and W. Wolf, Eds., Multiprocessor Systems-on-Chip. Morgan Kaufmannn, 2004.

[27] V. Aggarwal, I. A. Troxel, and A. D. George, "Design and Analysis of Parallel N-Queens on Reconfigurable Hardware with Handel-C and MPI," in 2004 MAPLD Intl. Conference, Washington, DC, USA, 2004.

[28] "OpenFPGA GenAPI version 0.4 Draft For Comment," http://www. openfpga.org/pages/Standards.aspx [Accessed: September, 2008].

[29] Intel, "Intel QuickAssist Technology AAL (White Paper)," http://download.intel.com/technology/platforms/quickassist/quickassist_ aal_whitepaper.pdf [Accessed: September, 2008].

[30] M. Jones, L. Scharf, J. Scott, C. Twaddle, M. Yaconis, K. Yao, P. Athanas, and B. Schott, "Implementing an API for Distributed Adaptive Computing Systems," in FCCM '99: Proc. of the Seventh Annual IEEE Symposium on Field-Programmable Custom Computing Machines, Washington, DC, USA, 1999, p. 222.

[31] N. Moore, A. Conti, M. Leeser, and L. King, "Vforce: An Extensible Framework for Reconfigurable Supercomputing," Computer, vol. 40, no. 3, pp. 39-49, March 2007.

[32] C. Comis, "A High-Speed Inter-Process Communication Architecture for FPGA-based Hardware Acceleration of Molecular Dynamics," Master's thesis, University of Toronto, 2005.

[33] MPI Parallel Environment, http://www-unix.mcs.anl.gov/perfvis/ software/MPE/index.htm [Accessed: Sept. 2008].

[34] O. Zaki, E. Lusk, W. Gropp, and D. Swider, "Toward scalable performance visualization with Jumpshot," High Performance Computing Applications, vol. 13, no. 2, pp. 277-288, Fall 1999.

[35] D. Nunes, M. Saldaña, and P. Chow, "A Profiler for a Heterogeneous Multi-Core Multi-FPGA System," in In Proceedings of the 2008 Intl. Conference on Field-Programmable Technology, Taipei, Taiwan, 2008.

[36] M. Saldaña, E. Ramalho, and P. Chow, "A Message-passing Hardware/Software Co-simulation Environment to Aid in Reconfigurable Computing Design using TMD-MPI," in In Proc. of the 5th Intl. Conf. on Reconfigurable Computing and FPGAs., Cancun, Mexico, 2008.

[37] Mentor Graphics, Corp., http://www.mentor.com/.

[38] D. Wang, "An Automated Flow to Generate Hardware Computing Nodes from C for an FPGA-Based MPI Computing Network," Engineering Science Undergraduate Thesis, University of Toronto, 2008. 